\title{
The care continuum with interprofessional oncology teams: Perspectives of patients and family
}

by Karine Bilodeau, Sylvie Dubois, and Jacinthe Pepin

\begin{abstract}
To accompany the individual diagnosed with cancer along the care continuum, teams of professionals have been created based on integrating an interprofessional patient-centred (IPPC) practice. The goal of this article is to present some of the results of a case study documenting IPPC practice carried on by teams within the oncology care continuum. Observations and interviews with patients and their family were conducted. The results suggest that IPPC practice is variable within teams, but optimal at the beginning of treatments or cancer recurrence. However, patients can experience breakdowns in the continuity of care and more difficult transitions between oncology continuum periods (diagnosis, treatment, follow-up).
\end{abstract}

Key words: multidisciplinary team, care continuum, cancer, patient-centred care, patient perspective

\section{INTRODUCTION}

C anadian policies promote accessibility to health services and the importance of providing people diagnosed with cancer with comprehensive care along the entire care continuum (Canadian Partnership Against Cancer, 2013). However, this population reports having to face fragmented and inaccessible care (Thorne \& Truant, 20I0). Interprofessional oncology teams represent a solution to facilitate the patients' journey along their continuum (Direction de lutte contre le cancer [DLCC], 2007). These teams provide services related to screening, investigation, treatment and rehabilitation, in addition to communicating relevant information and offering psychological, social and spiritual support (DLCC, 2007).

\section{ABOUT THE AUTHORS}

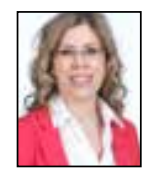

Karine Bilodeau, $\mathrm{RN}, \mathrm{PhD}, \mathrm{CON}(\mathrm{C})$, is completing a postdoctoral internship in the clinical science program at the University of Sherbrooke and is a Research Fellow at the Charles Lemoyne Hospital Research Centre. This study was completed as part of the doctorate study program in nursing science at the Universite de Montréal. Email: karine.bilodeau2@usherbrooke.ca

Address all correspondence to this author.

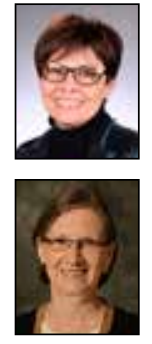

Sylvie Dubois, RN, PhD, is Director of Nursing at University of Montreal Health Centre (CHUM) and Associate Professor at the Faculty of Nursing, Université de Montréal.

Jacinthe Pepin, RN, PhD, is a Full Professor at the Faculty of Nursing, Université de Montréal, and Director, CIFI (Centre for Innovation in Nursing Education), Université de Montréal.

DOI: $10.5737 / 236880762513036$
Given that care to the individual with cancer may be restricted if it lacks continuity (Lewin, Skea, Entwistle, Zwarenstein, \& Dick, 200I), carrying out interprofessional patient-centred (IPPC) practice along the entire continuum appears to be necessary. Because of the varied types of expertise and contributions from each of the professionals, the individual with cancer can be presented through this practice with an integrated and non-fractured vision of their entire health situation (Legault, 2003). Incidentally, studies conducted with patients living with cancer indicate that they appreciate an open and respectful attitude from professionals (Kvale \& Bondevik, 2008), but lament not receiving information that meets their needs and not participating in shared decision-making (Sinfield, Baker, Agarwal, \& Tarrant, 2008). Further, Barnet and Shaw's (2013) literature review emphasizes that patients want special attention paid to their psychological health, a reduction in waiting times and the assignment of a resource person to answer their questions, such as a pivot nurse. According to these authors, the greatest challenge for interprofessional teams is to maintain care, from diagnosis to end of life, while making sure they keep on being centred on the patient. Despite the importance of engaging in IPPC practice, to our knowledge, few studies have documented this practice in the light of the oncology care continuum and patients' and families' perspectives.

A qualitative study of multiple cases was carried out as part of the doctoral research project by the first author. This study aimed to describe IPPC practice all along the oncology care continuum. The study sought to answer the following research question: How do teams engage in IPPC practice along the care continuum? To answer this question, it was important to describe the oncology care continuum and state the intensity with which this practice is being deployed with the patient and their family during each of the phases. The aim of this article is to present the research results that exclusively document the IPPC practice carried on by teams along the oncology care continuum especially regarding the experience of patients and their families.

\section{IPPC practice}

To support the description of IPPC practice, the Person Centred Nursing Framework (PCNF) of McCormack and McCance (2010) was adapted by integrating interprofessionality as defined by Couturier (2008) and was used as a framework. According to this adaptation of the PCNF, when engaged in IPPC practice, the team works with the patient's beliefs and values, is involved, demonstrates empathy, shares decision making with the patient and provides holistic care (Bilodeau, Dubois, \& Pepin, 2013). This practice is carried out in a space for mutual exchanges between professionals and patients and their loved ones. 
Table 1: Participants' characteristics

\begin{tabular}{|c|c|c|c|c|c|c|c|}
\hline \multicolumn{4}{|l|}{ CASE: Team A $(n=5)$} & \multicolumn{4}{|c|}{ CASE: Team B $(n=6)$} \\
\hline & Vladimir & $M$ & 74 & Diagnosis & Suzanne* & $F$ & 57 \\
\hline Treatment & Maria & $\mathrm{F}$ & 70 & Treatment & Cathie & $F$ & 23 \\
\hline & Jenny* & $\mathrm{F}$ & 26 & Recurrence & Janice & $\mathrm{F}$ & 59 \\
\hline \multirow[t]{2}{*}{ Follow-up } & \multirow[t]{2}{*}{ Béatrice } & \multirow[t]{2}{*}{$F$} & \multirow[t]{2}{*}{48} & \multirow[t]{2}{*}{ Follow-up } & Céline & $F$ & 53 \\
\hline & & & & & Marc* & $M$ & 52 \\
\hline
\end{tabular}

\section{The oncology care continuum}

In this study, the oncology care continuum is considered as comprising four periods: investigation, diagnosis, treatments and follow-up (Direction de la lutte contre le cancer, 20II). The investigation period is characterized by the appearance of cancer symptoms and the wait for screening and investigation tests results. The diagnosis period represents the communication of cancer diagnosis and the wait for treatments. Later on, the treatment period corresponds to the beginning of treatments or their modification in case of recurrence. Lastly, follow-up is characterized by the end of treatments and the transition to survivorship where medical follow-up and monitoring tests will need to be performed.

\section{METHOD}

A multiple case study (Stake, 2008) was conducted with two interprofessional oncology teams in order to describe IPPC practice. This type of study presents a rich, complex and holistic description of a phenomenon. For this research, the phenomenon under study was IPPC practice along the oncology care continuum. The cases were two interprofessional teams within a university medical centre (UMC) in an urban area of Quebec, each dedicated to a particular type of cancer that affects both men and women. After obtaining an ethics certificate from the clinical setting, teams were selected with the assistance of oncology managers. Purposeful sampling (Patton, 2002) was used to target participants. Inclusion criteria were fluency in French and an interest in participating in the study. As for patients, they also had to be diagnosed with cancer; fall within one of the care continuum periods unfolding at the medical centre, i.e., diagnosis, treatments including recurrence, and follow-up; be cared for at the UMC where the study was occurring, and be of a physical and mental condition assessed as stable by the referring professionals. No criteria clarifying physical and mental stability were given to the professionals assessing the eligibility of patients to participate in the study. Regarding loved ones, they had to accompany the patient and be older than I8 years of age. Professionals collaborated in the recruitment of patients and patients recruited loved ones.
The results of eight semi-structured interviews with patients $(n=5)$ sometimes accompanied by a loved one $(n=3)$ and of our observing these patients and loved ones were reported to describe IPPC practice teams along the care continuum. The interview questions explored the participants' experience with interprofessional teams and their perceptions regarding IPPC practice. The observations completed by the first author consisted in accompanying recruited patients during various activities with the team (e.g., appointments, treatments). The aim of the observations was to describe how the patients experienced IPPC practice. In all, 57.6 hours of observation were completed with the two teams. Table I illustrates the characteristics of patients and loved ones in the sample $(\mathrm{n}=\mathrm{II}) .{ }^{\mathrm{I}}$ The majority of participants were of Caucasian origin $(n=9)$. Fictitious names were assigned to participants.

In accordance to Stake (I995), the transcribed data (interviews and observations) were submitted to a content analysis via an iterative process including the following activities: abstracting, presentation of data, development and verification of findings (Miles \& Huberman, 2003). Furthermore, the cases were contrasted to describe their particularities. The QDA Miner v.4.o.II software was used to manage qualitative data. The selected scientific rigor criteria were credibility (authenticity and plausibility of the research), dependability (stability of data during analysis), procedural accountability (description of the research process) and transferability (knowledge transfer potential to the reader) (Guba \& Lincoln, I989). To meet these criteria, sustained observation of the teams, interrater validity, and interview and observation data triangulation were performed. Moreover, it is through a re-reading and abstracting of the data that we were able to validate the consistency of the findings being drawn. A journal was kept to document the first author's personal impressions when collecting data, collate methodological and interpretative notes in addition and keep track of the research process. Finally, the context for IPPC practice in oncology was documented in a detailed fashion.

I. The complete sample for the doctoral study included 8 patients, 3 loved ones, I 8 professionals and 2 managers $(\mathrm{N}=3 \mathrm{I})$. 


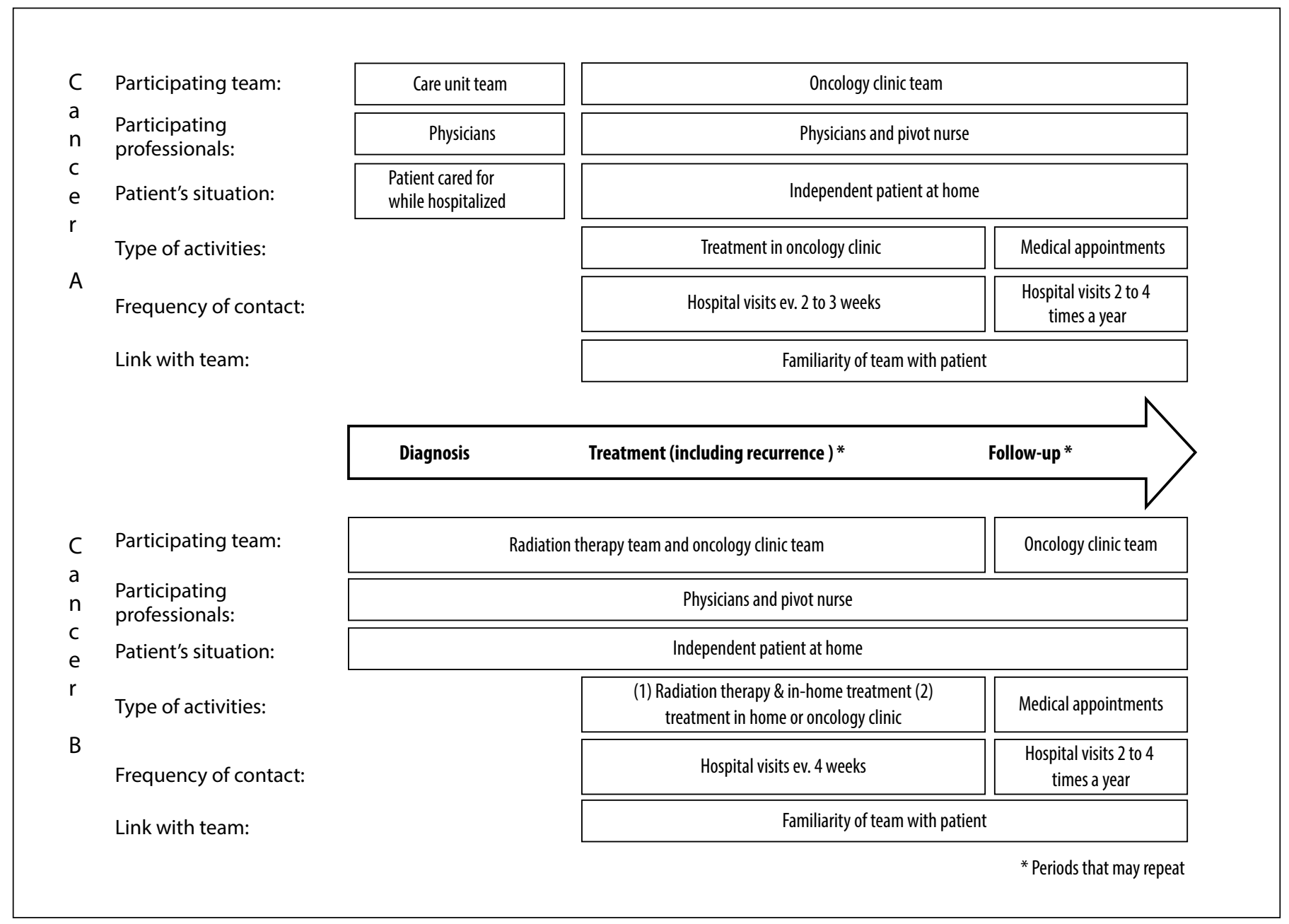

Diagram I: Involvement of interprofessional teams according to the oncology care continuum

\section{RESULTS}

The results presented herein related to the IPPC practice being carried on by two teams at various periods of the care continuum (diagnosis; treatments including recurrence; follow-up) as perceived by patients and their loved ones. This description is preceded by a brief presentation of case characteristics. Diagram I presents the involvement of the interprofessional teams within the oncology care continuum.

\section{Case characteristics}

Team A. Team A's patients had a cancer where the survival rate was below 5 years. The main treatments for this type of cancer were surgery and intravenous chemotherapy. Participants had to go to the hospital regularly for appointments and treatments. At data collection, patients reported being bothered by the chemotherapy side effects (e.g. nausea, asthenia). Team A's professionals ${ }^{2}$ comprised those who worked in the care unit and oncology clinic.

Team B. Team B's patients had a cancer where the survival rate was below 2 years. After a biopsy or surgery, a concurrent radiation therapy and oral chemotherapy treatment was provided to patients. At the end of this treatment, only the chemotherapy treatment was continued. Because of the treatment type, a lower number of medical appointments were required.
During the interviews, the patients and significant other's discourse was tinged with helplessness because of the losses and grief they were experiencing (e.g. holding a job, driving a car). Moreover, while dealing with treatments and appointments, the participants had to deal without help with several personal issues (e.g. mandate given in anticipation of incapacity, health insurance). Team B comprised those who worked in the care unit, radiation therapy clinic and oncology clinic.

\section{Diagnosis period}

During the diagnosis period, the participants had different opportunities for interaction with professionals depending on the team. Team A patients were in touch with the surgeon with whom a strong trusting relationship appeared to have been established from communication of diagnosis. As Béatrice explained: "He had my life in his hands so I had to be trusting." This period essentially unfolded with the care unit professionals. The observation revealed that the team's activities were directed at perioperative care. Regarding Team $\mathrm{B}$, patients were briefly hospitalized for a biopsy or surgery.

2. Various health care professionals made up Teams A and B, for example, pivot nurse, physician, physiotherapist, nutritionist, psychologist, pharmacist, social worker or occupational therapist. 
Afterwards, the diagnosis was announced by one of the physicians at interprofessional rounds which were attended by physicians representing various specialties and the pivot nurse, all of whom were presented to the patients.

The diagnosis period is characterized by the shock associated with the communication of the cancer and the need to quickly make a decision regarding treatments. The majority of participants stressed they had received a lot of information. Regarding Team A, all patients acknowledged receiving information that was clear and in sufficient quantity. As Diane commented: "When he explained to me he was going to operate me for my tumour, he drew me a picture (...) because he could see I would not understand anything." Regarding Team $\mathrm{B}$, some patients mentioned not having been receptive to the large quantity of information received over a short time from many professionals. They added they would have appreciated a pause before being given information regarding treatments because they were not receptive then. As Céline shared: "A visit with five, six [professionals], right away (...), I did not listen to them (...) I was so taken aback by the announcement that had been made to me [diagnosis]."

Patients had to make choices quickly concerning their treatments as their cancers (A and B) were progressing rapidly. The majority of Team A patients expressed how ambivalent they felt about the treatment-related choices. As Maria said: "But I told him [the physician] too 'At what cost?' [accepting chemotherapy]. I am a very independent person and I don't want to see myself all ragged." Regarding Team B's patients, they reported having no choice but to accept the treatments if they wanted to survive. As Cathie stated: "There was not really any decision to be made by me (...) it was prompted [by professionals]: you're going to get chemo, you're going to get radiation therapy."

\section{The treatment period including recurrence}

Throughout this period, most of the participants for both teams appreciated the "smiles" and "sensitivity" of professionals to their situation. Béatrice gave this explanation: "Everywhere, I have always been received with much friendliness and kindness." Furthermore, patients were no longer hospitalized and had to travel to meet with their team. They were autonomous and were making their decision. As summed up by Béatrice:

"It might be me who is not in the same position. In one place, I am bed-ridden [care unit], in the other, I am standing [oncology clinic]. (...) I was much more in control of what was happening for the chemotherapy [oncology clinic]." (Béatrice)

Services were organized differently by each team. Regarding Team A, the follow-up was performed by professionals in the oncology clinic. The pivot nurse was introduced to the patients. Nevertheless, patients admitted staying in touch with the surgeon they had met during the diagnosis period. Three of them made new appointments with him to discuss chemotherapy treatments even though he was no longer their attending physician. As for Team B, patients had already had a brief meeting with professionals at communication of diagnosis. As treating cancer B first required a concurrent treatment with oral chemotherapy and radiation therapy, the services were provided in the radiation therapy clinic on that occasion. At the end of this concurrent treatment, patients moved to the oncology clinic. Thus, they had to adapt to this change in environment. As Suzanne explained: "When you come out of radiation oncology, it's like you are left to your own devices.”

When a cancer recurrence is announced, it was reported by patients and observed during data collection that both teams made every effort to accompany patients. The latter were often already known to the teams and this enabled the personalization of services. When the recurrence of her cancer was communicated to her, Diane appreciated that her history was known, that empathy was demonstrated to her and that she was given special attention. As she explained: "It's the same team, it's comforting (...) because when I came back, I did not have to explain my case once again (...) They were already aware of it."

Most of the time, patients for both teams met individually with the professionals. However, the frequency of meeting with the teams was different. As Team A patients travelled regularly to the hospital, they could cross paths with professionals such as the pivot nurse or the pharmacist. Several professionals were close to the locations attended by patients. As Jenny said: "During the first series [of chemotherapy treatments], we would see her [the pivot nurse] nearly every time anyway." Therefore, patients had more opportunities to ask questions and exchange with them. Team B patients would come only once or twice a month to the hospital centre for their medical appointments. They rarely met with other professionals except for the pivot nurse; they could phone her with any question they may have. As Paul stated: "If one had problems, in general, one would talk to the pivot nurse [on the phone]."

Team A patients reported that they felt the team worked in synergy because they would sometimes get evidence that the professionals were collaborating. Patients noticed that professionals reported what other professionals had said or told them directly having consulted with a colleague. As Diane indicated:

"You can see there's communication, because, at times, they talk to you about something that's probably none of their business (...) but they know about it (...) I find this reassuring." (Diane)

It is worth mentioning that the Team B patients stressed they had not noticed any team work, even though the available professionals and services had been introduced to them at communication of diagnosis. As Marc confided: "We said to ourselves: things are pretty bad [cancer diagnosis], but we have a nice team and we'll get plenty of services. Afterwards (...) it was up to us to deal with that [obtaining services]." (Marc)

Despite the existence of interprofessional teams, interventions were neither necessarily faster nor continuing for the two teams' patients. It was also observed that the professionals might not take account of the patients' opinion and experience. Some patients reported they "imposed themselves" on the team either by participating in their own care (e.g., 
keeping a diary of their symptoms) or by refusing some care. Furthermore, breakdowns were reported and observed in the continuity of their care such as an unorganized appointment, having to retake a test because the information had not been entered in the chart or waiting for an intervention. Patients had to be vigilant and attentive to receive adequate care. An observation memo referred to this situation:

Janice indicates that while talking on the phone with the pivot nurse, she realized she had not followed the usual instructions for taking her chemotherapy. She had not been given the instructions sheet. (Memo-Janice)

Team B participants reported not having received enough information during the treatment period. Patients and their loved ones admitted they did not understand the procedures related to treatments. They felt they had been left hanging. Even though Team B cancer had a poor prognosis, the patients and their families wanted to obtain detailed information on the matter. As Cathie explained:

It took time before I asked the question [about when I was going to die] and I feared the answer. But I was not told (...) what my life expectancy was. I did not know if I was buying myself a few more years (...) because we don't know how long I am going to take it [the treatment](...) what is next? (...) what are the signs that it [the cancer] is coming back or not coming back?" (Cathie)

\section{The follow-up period}

During the follow-up period, visits to the oncology clinic were rare (three to four times a year). Patients for both teams indicated how much they appreciated being able to contact the pivot nurse with any of their questions because they feel there is "always a sword hanging over their heads." This nurse was the access point to the team even though, as was highlighted by Céline, most of the services were offered and explained during the diagnosis period, it was at the end of the treatments that needs are felt the most. As she stated:

"I said, after 14 months of chemo, I think I may well be entitled to a few of the [psychology] sessions I did not use during the first year (...) you go when you are ready. It was of no use for me to go or meet with you because I would not have talked then [diagnosis period]." (Céline)

Finally, this period is characterized by the desire to obtain support when deemed appropriate by the patients. The next section is the discussion of the results.

\section{DISCUSSION}

When engaging in IPPC practice, the health team works with the values of the patient, gets involved, shows empathy, offers shared decision making and holistic care. However, the results of our study reveal variations in the practices. To the best of our knowledge, few studies have documented this type of practice according to the oncology care continuum and patients/significant others' perspectives. It was also found that the sharing of information between professionals was sporadic, especially during the diagnosis period at which time patients reported they wanted to obtain exact and complete information when they feel receptive. The results also show that it is during the treatment period that patients notice the professionals' people skills (welcome, friendliness), but it is when the cancer recurs that IPPC practice appears to be optimal. This may be explained by the fact that the team already knows the patient, this familiarity with the patient seems to enhance involvement in IPPC practice.

Describing the intensity of IPPC practice by health teams along the continuum is an original contribution from the present study. During the diagnosis period, a relationship of trust seemed to be more developed with the physician than with any other members of the interprofessional team. It is during the treatment and recurrence period that the participants had the most contact with the team at which time they could notice the interprofessional nature of the work. Concerning the follow-up period, results highlight the patients' desire to access support besides that of the pivot nurse, and their difficulties in obtaining professional services (e.g., psychologist). Several authors report that during this period patients are faced with many issues and concerns (e.g., fatigue, anxiety, fear of recurrence) necessitating care and services (Alfano, Ganz, Rowland, \& Hahn, 20I2; Ness et al., 20I3). This study's results suggest that interprofessional teams are more active with the patients during the treatment and recurrence period. However, patients want the interprofessional follow-up to be centred on the patient where professionals do adjust their practice according to the particularities experienced within the care continuum.

The results from interviews and observations with patients also show that they rarely witness collaboration between team members. Observations revealed that Team B patients had few contacts with professionals because of oral chemotherapy being taken at home. Patients then have fewer opportunities for interaction, assessment and training with team members (Given, Spoelstra, \& Grant, 20II; Simchowitz et al., 20I0). This could explain why patients reported not noticing interprofessional work regarding Team B as opposed to Team A. Each team's care setting becomes an important element that may influence IPPC practice (e.g., service organization, treatment modalities). Thus, to appreciate IPPC practice, it seems important that patients experience regular contacts with the team members, depending on the health care setting.

Results also revealed that IPPC practice was variable, as breakdowns in continuity were reported and observed with both teams. As indicated by several authors (Barnet \& Shaw, 20I3; Mazor et al., 20I2), breakdowns in continuity related to delays, information sharing, care coordination and support are all elements that have a negative influence on the care continuum. It is worth noting that for several years, many governmental initiatives have been developed in order to encourage the implementation of pivot nurses (Direction de la lutte contre le cancer, 2008) or patient navigators (Institute of Medicine, 20II) to facilitate the coordination of care and alleviate the burdens associated with the lived experience of patients diagnosed with cancer (e.g., anxiety, financial problems). Even though the patients of the two teams under study had access to a pivot nurse and stated they appreciated his/her services, it seems that it is not sufficient to ensure continuity of care. It is, 
then, possible to believe that continuity of care is an indicator of quality of care in the context of IPPC practice.

Moreover, the study results suggest the patients lived through periods of vulnerability, also called "critical transition" (Clausen et al., 20I2), which are characterized by a reduction in the contacts with certain professionals when transitioning from one period of the care continuum to another (e.g., diagnosis and beginning of treatments). On the one hand, Team A patients did not have access to a pivot nurse to facilitate their transfer to the oncology clinic, but they referred themselves to their physician. On the other hand, Team B participants had access to a pivot nurse from the moment the diagnosis was announced, but her presence did not seem to facilitate the transition from the radiation therapy clinic to the oncology clinic. These results suggest that interprofessional oncology teams do not provide totally integrated care along the care continuum. It would thus appear that a smooth transition between the various periods of the care continuum is evidence of an optimal IPPC practice (Walsh et al., 20IO).

There are some limits to our study. Let's mention first that observation periods were less frequent for patients going through the follow-up period because of their less-frequent visits to the oncology clinic. This situation may have affected the richness of the description of IPPC practice during that period. Also, one cannot overlook the fact that the first author who completed interviews and observations was known to the professionals; this may have influenced the unfolding of certain interactions with both patients and professionals. It is also appropriate to specify that the results of the present study are connected with a particular context, that of oncology. Any use of these results must call for a similar application context.

\section{Implication for practice and research}

These results point out that IPPC practice varies in its intensity depending on the care continuum periods and each team's specific setting. They also reveal a need patients

\section{REFERENCES}

Alfano, C.M., Ganz, P.A., Rowland, J.H., \& Hahn, E.E. (20I2). Cancer survivorship and cancer rehabilitation: Revitalizing the link. Journal of Clinical Oncology, 30(9), 904-906.

Barnet, M., \& Shaw, T. (2013). What do consumers see as important in the continuity of their care? Supportive Care in Cancer, 21(9), 2637-2642.

Bilodeau, K., Dubois, S., \& Pepin, J. (2013). Contribution des sciences infirmières au développement des savoirs interprofessionnels. Recherche en Soins Infirmiers, II3, 43-50.

Canadian Partnership Against Cancer. (2013). Improving the Cancer Journey 2013, from http://www.partnershipagainstcancer.ca/ priorities/cancer-journey/

Clausen, C., Strohschein, F.J., Faremo, S., Bateman, D., Posel, N., \& Fleiszer, D.M. (20I2). Developing an interprofessional care plan for an older adult woman with breast cancer: From multiple voices to a shared vision. Clinical Journal of Oncology Nursing, 16(I), EI8-25.

Couturier, Y., Gagnon, D., Carrier, S., \& Etheridge, F. (2008). The interdisciplinary condition of work in relational professions of the health and social care field: A theoretical standpoint. Journal of Interprofessional Care, 22(4), 34I-35I. experience during the follow-up period. It would thus be important to: I) identify a patient navigator from the diagnosis on; 2) provide relevant information according to the care continuum period; 3 ) identify with the patient the critical transition periods and give these careful consideration; and 4) maximize the contact frequency with the patient and family. Furthermore, interprofessional teams ought to be sensitive to the follow-up after the cancer experience and to breakdowns in continuity all along the care continuum, because these have a negative influence on the cancer experience. It is then critical for the teams to optimize their IPPC practice.

To our knowledge, few research projects have chosen to study IPPC practice from the perspective of patients and their families using a multiple case study with a qualitative design. With this design, we were able to describe the particularities of two health care teams with different settings. Further research is necessary to document IPPC practice along the entire care continuum for each type of cancer so that recommendations can be issued for the accompaniment of patients. It is also suggested to consider patient and family observation as a relevant, complementary research strategy to document in detail particularities of the oncology care continuum.

\section{ACKNOWLEDGEMENTS}

We would like to recognize the financial support from the Foundation of the Université de Montréal Hospital Centre (CHUM), CHUM's Nursing Administration, Quebec's MELS (Ministry of Education, Leisure and Sports), the Faculty of Nursing Science (FSI), Université de Montréal, Faculty of Graduate and Postdoctoral Studies (FESP), Université de Montréal, the Quebec Nursing Intervention Research Network (RRISIQ), Canadian Nurses Foundation (CNF), CHUM's Research Centre (CRCHUM) and the Canadian Association of Nurses in Oncology (CANO/ACIO).

Direction de la lutte contre le cancer. (2008). Rôle de l'infirmière pivot en oncologie. Québec Ministère de la santé et des services sociaux.

Direction de la lutte contre le cancer. (2007). Orientations prioritaires 2007-2012 du programme québécois de lutte contre le cancer. Québec: MSSS du Québec.

Direction de la lutte contre le cancer. (20II). Rapport du comité d'oncologie psychosociale: Vers des soins centrés sur la personne. Québec: MSSS.

Given, B.A., Spoelstra, S.L., \& Grant, M. (20II). The challenges of oral agents as antineoplastic treatments. Seminars in Oncology Nursing, 27(2), 93-IO3.

Guba, E.G., \& Lincoln, Y.S. (1989). Fourth generation evaluation. Newbury Park: Sage Publications.

Institute of Medicine. (20II). Patient-centered cancer treatment planning: Improving the quality of oncology care: Workshop summary. Washington: National Academies Press.

Kvale, K., \& Bondevik, M. (2008). What is important for patient centred care? A qualitative study about the perceptions of patients with cancer. Scandinavian Journal of Caring Sciences, 22(4), 582-589. 
Legault, G.A. (2003). Crise d'identité professionnelle et professionnalisme. Québec: Presses de l'Université du Québec.

Lewin, S., Skea, Z.C., Entwistle, V., Zwarenstein, M., \& Dick, J. (200I). Interventions for providers to promote a patient-centred approach in clinical consultations. Cochrane Database Syst Rev(4), CDoo3267.

Mazor, K.M., Roblin, D.W., Greene, S.M., Lemay, C.A., Firneno, C.L., Calvi, J., et al. (20I2). Toward patient-centered cancer care: Patient perceptions of problematic events, impact, and response. Journal of Clinical Oncology, 30(I5), I784-I790.

McCormack, B., \& McCance, T.V. (2010). Person-centred nursing: Theory and practice. Oxford: John Wiley and Sons.

Miles, M.B., \& Huberman, A.M. (2003). Analyse de données qualitatives (2e ed.). Paris: De Boeck Université.

Ness, S., Kokal, J., Fee-Schroeder, K., Novotny, P., Satele, D., \& Barton, D. (2013). Concerns across the survivorship trajectory: Results from a survey of cancer survivors. Oncology Nursing Forum, 40(I), $35-42$.

Patton, M.Q. (2002). Qualitative research Q evaluation methods (3rd ed.). Thousand Oaks, Calif.: Sage Publications.
Simchowitz, B., Shiman, L., Spencer, J., Brouillard, D., Gross, A., Connor, M., et al. (20I0). Perceptions and experiences of patients receiving oral chemotherapy. Clinical Journal of Oncology Nursing, 14(4), 447-453.

Sinfield, P., Baker, R., Agarwal, S., \& Tarrant, C. (2008). Patientcentred care: What are the experiences of prostate cancer patients and their partners? Patient Education and Counseling, 73(I), 9I-96.

Stake, R.E. (1995). The art of case study research. Thousand Oaks: Sage Publications.

Stake, R.E. (2008). Qualitative case studies. In N.K. Denzin \& Y.S. Lincoln (Eds.), Strategies of qualitative inquiry (3rd ed., pp. II9I49). Los Angeles: Sage Publications.

Thorne, S., \& Truant, T. (20I0). Will designated patient navigators fix the problem? Oncology nursing in transition. Canadian Oncology Nursing Journal 20(3), II6-I2I.

Walsh, J., Harrison, J.D., Young, J.M., Butow, P.N., Solomon, M.J., \& Masya, L. (2010). What are the current barriers to effective cancer care coordination? A qualitative study. BMC Health Services Research, 10, I32. 